\title{
Effects of emotional reactivity on inhibitory avoidance in the elevated T-maze
}

\section{Conde ${ }^{1}$, \\ V. Costa ${ }^{1}$ and \\ C. Tomaz ${ }^{1,2}$}

\section{Correspondence}

C. Tomaz

Laboratório de Neurobiologia

Universidade de Braślia

Caixa Postal 04631

70910-900 Brasília, DF

Brasil

Fax: + 55-61-274-1251

E-mail: ctomaz@unb.br

Research supported by CN Pq and FAPESP. C. Conde was the recipient of a PhD fellowship from Colciencias and Universidad Industrial de Santander (Colombia), and CNPq. V. Costa was the recipient of an undergraduate fellowship from CNPq.

Received December 21, 1998 Accepted N ovember 30, 1999
${ }^{1}$ Departamento de Fisiologia and Laboratório de Psicobiologia, Faculdade de Filosofia, Ciências e Letras de Ribeirão Preto, Universidade de São Paulo, Ribeirão Preto, SP, Brasil 'Laboratório de Neurobiologia, Universidade de Brasília, Brasília, DF, Brasil

\section{Abstract}

The possibility of the presence of inter-individual emotional differences and the memory performance of rats was examined in the elevated T-maze. Two kinds of aversively motivated behaviors, inhibitory avoidance and escape learning, were measured. Based on the number of trials to achieve a learning criterion, rats were divided into two subgroups with either low or high avoidance reactivity (LAR or HAR, respectively). Retention test avoidance latencies showed that HAR animals had better avoidance memory (Mann-Whitney rank sum test, $\mathrm{P}=0.0035)$. No such differences were found for the escape component of this test. These data suggest that individual emotional differences affect inhibitory avoidance performance, which may help to explain the dispersion of the data observed in other studies using this paradigm.

A large number of studies have described strains of rats selected on the basis of their emotional reactivity and conditionability. In general, these strains are the outcome of bidirectional selection for emotionality differences determined by open-field defecation and ambulation scores, as well as the speed of acquisition and retention of a conditioned avoidance response measured in an escapeavoidance conditioning apparatus. Examples are the Maudsley rats (1), Roman rats (2), Naples rats (3), Syracuse and Australian rats (4), and some selections of Sprague-Dawley rats (5).

Recently, Graeff and co-workers (6-8) described the elevated T-maze test as a new method for investigating emotionally related

\section{Key words}

- Elevated T-maze

- High and low

avoidance rats

- Anxiety

- Emotional reactivity behaviors and processes underlying learning. The apparatus is composed of two open arms arranged at right angles to one enclosed arm, elevated above the ground. The maze permits the measurement of two kinds of aversively motivated behaviors, namely, inhibitory avoidance (time the animal takes to leave the enclosed arm) and one-way escape (time taken to leave the open arm). This experimental model allows the parallel measurement of responses related to both innate and learned fear in the same subject, and permits the simultaneous assessment of memory for these behaviors. The elevated Tmaze has been shown to be sensitive to the effects of anxiolytic and memory-modulating drugs such as diazepam, the 5- $\mathrm{HT}_{1 \mathrm{~A}}$ ligand 
ipsapirone $(6,7,9)$ and the $5-\mathrm{HT}_{3}$ receptor antagonist BRL 46470A (10). Moreover, the results of these studies suggest that the two tasks measured in the T-maze test (inhibitory avoidance - conditioned component, and oneway escape - unconditioned component) generate distinct types of fear and memory that could be related to different kinds of psychiatric disorders and thus be differently affected by distinct drugs.

Although the results of these studies are promising, an examination of the data for the conditioned component indicates a wide dispersion of measured values. This dispersion is not likely to be due to animal manipulation by the experimenter. In a recent study we observed a similar dispersion in rats tested in the T-maze using a free exploration paradigm, i.e., animals were not handled during the experimental sessions (Echeverry MB, Hasenhöhrl R, Huston JP and Tomaz C, unpublished results). Therefore, it is possible that such dispersion could indicate individual differences in emotional reactivity. If this is the case, the conditioning performance in the T-maze may reflect the emotionality profiles of the subjects, which could then be selected on the basis of number of training trials required to achieve a learning criterion. The present study was undertaken to examine this possibility using a learningto-criterion procedure.

Male Wistar rats (weight, 250-230 g) obtained from the animal colony of the Ribeirão Preto Campus, University of São Paulo, were housed in groups of 4-6 and maintained under controlled temperature (22 $\pm 1{ }^{\circ} \mathrm{C}$ ), with a 12 -h light/dark cycle (lights on 7:00). Food and water were freely available. The elevated T-maze apparatus was made of wood and had three arms of equal dimensions $(50 \times 10 \mathrm{~cm})$. One arm, enclosed by $40-$ $\mathrm{cm}$ high walls, was perpendicular to the two opposite open arms. The whole apparatus was elevated $40 \mathrm{~cm}$ above the floor. The animals were handled gently for 5 min for 3 6 days after their arrival in the laboratory.
Training and testing in the elevated T-maze were as follows. On the first day, each rat was placed at the end of the enclosed arm facing the intersection of the arms, and the time taken to leave this arm with the four paws was recorded (baseline latency). The animal was then immediately removed from the maze and the same measurement was repeated (60-s intervals) for as many trials as needed for the rat to stay in the enclosed arm continuously for $300 \mathrm{~s}$ (learning criterion for the avoidance conditioning). After another $60 \mathrm{~s}$, the rat was placed at the end of the right open arm, and the time taken to leave this arm with the four paws was recorded (escape learning). Three days later, avoidance and escape latencies were measured again in one trial.

Using the number of trials required to achieve the criterion of 300 continuous seconds in the enclosed arm as a "reactivity indicator", the animals were divided into two groups: "high avoidance reactivity rats" (HAR), that needed three or less trials, and "low avoidance reactivity rats" (LAR), that needed more than three trials.

The correlation between the number of trials to reach the criterion and the corresponding test trial values was statistically significant $(\mathrm{r}=-0.472, \mathrm{P}=0.002, \mathrm{~N}=41$; Spearman rank order correlation), i.e., HAR showed better retention performance than LAR. Test latencies for the inhibitory avoidance component were higher than baseline values for both the HAR and LAR groups $(\mathrm{P}<0.001$; Wilcoxon signed rank test). However, when comparing the test avoidance latencies of HAR and LAR we observed that HAR latencies were higher $(\mathrm{P}=0.0035$; Mann-Whitney rank sum test; see Figure 1 A). These results suggest a close relationship between "reactivity level" and memory performance for the avoidance component in the elevated T-maze test.

Analysis of escape behavior does not indicate such a difference between HAR and LAR. There was no significant difference 
( $\mathrm{P}<0.05$; unpaired Student $t$-test) between the two groups in the time taken to leave the open arm during the retention trial. Moreover, both groups showed a significant latency decrease between training and test trials $(\mathrm{P}<0.05$; paired Student $t$-test; see Figure 1B) indicating good memory performance for this component of the elevated T-maze.

The results of the present study suggest that Wistar rats from our animal colony are heterogeneous in terms of parameters associated with inhibitory avoidance in this protocol. In contrast, in the same animals, escape behavior seems to be associated to another phenomenon or another kind of mechanism, which is homogeneous in this population. In fact, the elevated T-maze has been proposed (6-8) for the measurement of two kinds of aversively motivated behaviors that could be related to different types of fear: anticipatory anxiety and innate fear. The inhibitory avoidance behavior could represent a generalized anxiety disorder, while the one-way escape could be related to phobia or panic disorder, since escape from the open arm of the maze is a response seemingly motivated by species-typical fear of openness and elevation. It has also been demonstrated that these two behaviors in this protocol are affected differently by distinct classes of drugs, suggesting different brain mechanisms involved in their control $(6,7,9)$. The present findings provide additional empirical support for this hypothesis. The results of the avoidance conditioning retention trial indicate that this measure of memory is influenced by emotional factors. HAR rats, which learned the task to criterion in three trials or less, showed a better retention performance than the LAR rats, which needed 4-13 trials to reach the learning criterion. Several studies have pointed out that anxiety and negative reinforcement memory are closely linked (11-13). It has been suggested that inter-individual neuroendocrine and neurochemical characteristics are associated with behavioral differences observed

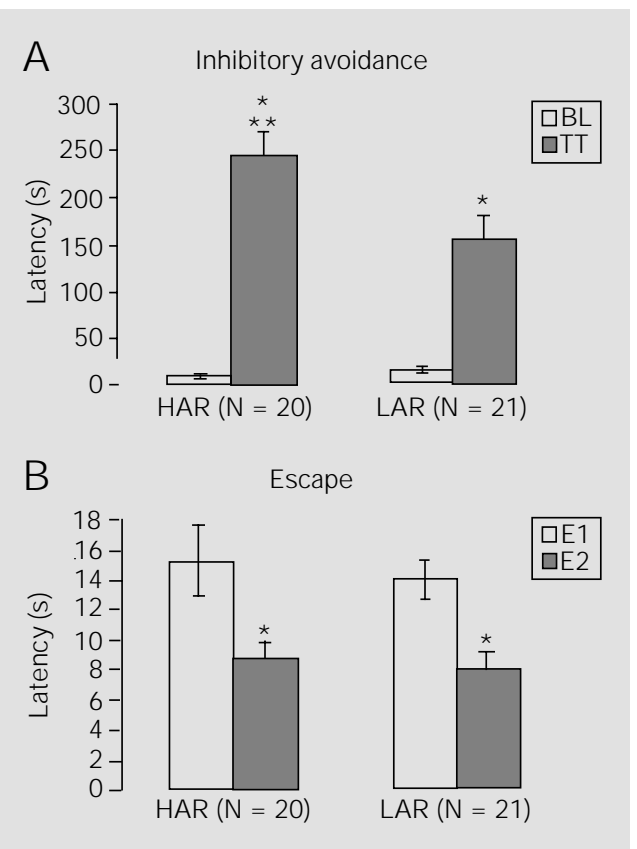

Figure 1 - Exit latency from the enclosed (A) and the open arms (B) in the elevated T-maze. BL = Baseline latency (first avoidance latency), $\Pi \mathrm{T}=$ test trial latency measured $72 \mathrm{~h}$ after the first exposure to the maze, HAR = high avoidance reactivity, $L A R=$ low avoidance reactivity, E1 = escape latency measured immediately after inhibitory avoidance training, E2 = escape latency measured $72 \mathrm{~h}$ later. Data are reported as means \pm SEM $* P<0.001$ compared with $B L$ values (A) and $* \mathrm{P}<0.01$ compared with E1 values (B) (Wilcoxon signed rank test). $* * \mathrm{P}=0.0035$ compared with TT LAR values (A) (Mann-Whitney rank sum test). in tests of anxiety and memory $(14,15)$. The possible nature of this difference in the elevated T-maze has not been investigated. Further neurobiological studies are important for a better understanding of these mechanisms since they might be related to human syndromes like anxiety, mood and cognitive disorders. Additionally, experiments including physiological measurements are important to corroborate our suggestion that animals reaching the criterion in three trials or less are more emotional reactive. Nevertheless, the present data show that investigators should account for possible inter-individual differences in terms of avoidance performance when using the elevated T-maze protocol. The use of a learning-to-criterion procedure (see also 16), as employed in the present study, could be useful to select rat subpopulations regarding emotional reactivity and conditionability.

\section{Acknowledgments}

We are grateful to José R. Stella for technical assistance. We thank C.M. Thiel for commenting on an early version of this article. 


\section{References}

1. J ay J r GE (1963). Genetic strains and stocks. In: Burdette WJ (Editor), Methodology in Mammalian Genetics. HoldenDay, San Francisco.

2. Bignanmi G (1965). Selection for high rates and low rates of avoidance conditioning in the rat. Animal Behavior, 13: 221-227.

3. Cerbone A, Pellicano M \& Sadile A (1993). Evidence for and against the Naples highand low-excitability rats as genetic model to study hippocampal functions. Neuroscience Biobehavior Reviews, 17: 295303.

4. Brush FR (1991). Genetic determinants of individual differences in avoidance learning: Behavioral and endocrine characteristics. Experientia, 47: 1039-1050.

5. Cure EM \& Rolinat J (1992). Behavioral heterogeneity in Sprague-Dawley rats. Physiology and Behavior, 51: 771-774.

6. Graeff FG, Viana M \& Tomaz C (1993). The elevated $T$ maze, a new experimental model of anxiety and memory: Effect of diazepam. Brazilian J ournal of Medical and Biological Research, 26: 1-4.

7. Viana MB, Tomaz C \& Graeff FG (1994).
The elevated T-maze: A new animal model of anxiety and memory. Pharmacology, Biochemistry and Behavior, 49: 549554.

8. Zangrossi H \& Graeff FG (1997). Behavioral validation of the elevated T-maze, a new animal model of anxiety. Brain Research Bulletin, 44: 1-5.

9. Graeff FG, Viana MB \& Mora PO (1996). Opposed regulation by dorsal raphe nucleus 5-HT pathways of two types of fear in the elevated T maze. Pharmacology, Biochemistry and Behavior, 53: 171-177.

10. Gargiulo PA, Viana MB, Graeff FG, De Sousa Silva MA \& Tomaz C (1996). Effects on anxiety and memory of systemic and intra-amygdala injection of 5-HT3 receptor antagonist BRL 46470A. Neuropsychobiology, 33: 189-195.

11. Beuzen A \& Belzung C (1995). Link between emotional memory and anxiety states: A study by principal component analysis. Physiology and Behavior, 58: 111-118.

12. Tomaz C, Brandão ML \& Garcia-Cairasco N (1992). Overlapping neural substrates underlying defense reactions, aversive memory, and convulsive behavior. In: Butcher LL, Decker M \& Lewin E (Editors), Neurotransmitter Interactions and Cognitive Functions. Birkhäuser, Boston.

13. Tomaz C, Dickinson-Anson $\mathrm{H}, \mathrm{McGaugh}$ J L, De Souza Silva MA, Viana MB \& Graeff FG (1993). Localization in the amygdala of the amnestic action of diazepam on emotional memory. Behavioural Brain Research, 58: 99-105.

14. Schwarting RKW, Thiel CM, Müller CP \& Huston J P (1998). Relationship between anxiety and serotonin in the ventral striatum. NeuroReport, 9: 1025-1029.

15. Steimer T, La Fleur S \& Schulz PE (1997). Neuroendocrine correlates of emotional reactivity and coping in male rats from the Roman high (RHA/Nerh)- and low (RLA/ Verh)-avoidance rats. Behavior Genetics, 27: 503-512.

16. Conde CA, Costa V \& Tomaz C (1999). Measuring emotional memory in the elevated T-maze using a training-to-criterion procedure. Pharmacology, Biochemistry and Behavior, 63: 63-69. 